\title{
Elevated Nectin-2 expression is involved in esophageal squamous cell carcinoma by promoting cell migration and invasion
}

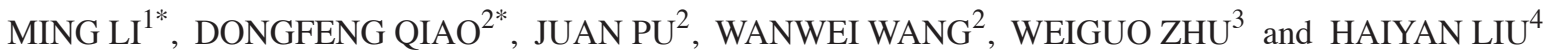 \\ ${ }^{1}$ Department of Radiotherapy Pathology, People's Hospital of Xuyi County, Huai'an, Jiangsu 211700; ${ }^{2}$ Department of \\ Radiotherapy Pathology, People's Hospital of Lianshui County, Huai'an, Jiangsu 223400; Departments of ${ }^{3}$ Radiotherapy \\ Oncology and ${ }^{4}$ Pathology, Huai'an First People's Hospital, Nanjing Medical University, Huai'an, Jiangsu 223300, P.R. China
}

Received December 15, 2015; Accepted June 15, 2017

DOI: $10.3892 / \mathrm{ol} .2018 .7953$

\begin{abstract}
Nectin-2 is overexpressed in cancer cells and is associated with poor prognosis in patients with various types of cancers. However, its involvement in esophageal squamous cell carcinoma (ESCC) remains unknown. The present study aimed to investigate the expression pattern of Nectin-2, its clinical significance and its roles in the malignant phenotypes of ESCC. Expression levels of Nectin-2 mRNA and protein were respectively detected by reverse transcription-quantitative polymerase chain reaction, western blotting and immunohistochemistry, based on 106 newly diagnosed ESCC patients. The associations between Nectin-2 expression and clinicopathological characteristics of ESCC patients were statistically analyzed. The effects of Nectin-2 in migration and invasion were then determined by wound healing and Transwell assays performed using ESCC cell lines (ECA109 and KYSE510) transfected with small interfering (si) RNA against Nectin-2. It was found that Nectin-2 expression was significantly elevated at the mRNA and protein levels in ESCC tissues, compared with the normal esophageal mucosa $(\mathrm{P}<0.001)$. Nectin-2-positive immunoreactivity was mainly localized in the cytoplasm of cancer cells in ESCC tissues. In addition, the expression levels of Nectin-2 protein in ESCC tissues with advanced tumor stage $(\mathrm{P}=0.006)$ and poor differentiation $(\mathrm{P}=0.02)$ were increased compared with patients with early tumor stage and well to moderate differentiation.
\end{abstract}

Correspondence to: Professor Haiyan Liu, Department of Pathology, Huai'an First People's Hospital, Nanjing Medical University, 6 Beijing West Road, Huai'an, Jiangsu 223300, P.R. China E-mail:1hyjsha@hotmail.com

Dr Weiguo Zhu, Department of Radiotherapy Oncology, Huai'an First People's Hospital, Nanjing Medical University, 6 Beijing West Road, Huai'an, Jiangsu 223300, P.R. China

E-mail: jshazwg@126.com

${ }^{*}$ Contributed equally

Key words: Nectin-2, esophageal squamous cell carcinoma, progression, migration, invasion
Additionally, knockdown of Nectin-2 in the 2 ESCC cell lines could effectively suppress the cell migration and invasion abilities $(\mathrm{P}<0.05)$. In conclusion, these findings revealed that Nectin-2 is generally overexpressed in ESCC and associated with aggressive cancer progression. The present data also indicated that the silencing of Nectin-2 with siRNA in ESCC cells may inhibit cell malignant biological properties, indicating its potential as a potential marker or a therapeutic target for ESCC.

\section{Introduction}

As one of the major types of esophageal cancer, esophageal squamous cell carcinoma (ESCC) is one of the most aggressive malignancies of the digestive tract, with high cancer-associated morbidity and mortality worldwide, due to the late diagnosis at advanced stages and the lack of effective treatment $(1,2)$. ESCC occurs mostly in developing countries (3). ESCC is characterized by early lymphatic and hematogenous metastasis, which is the main cause of the poor prognosis in patients with this malignancy (4). Among the available prognostic factors for ESCC, the International Union Against Cancer (UICC) tumor-node-metastasis stage has been recognized as the most important factor, since staging is determined by the involvement of lymph nodes, depth of invasion and presence of distant metastasis (5). However, the prognostic value of the stage is often affected by the heterogeneity of ESCC patients. Therefore, there is an urgent requirement to clarify the molecular mechanisms that drive the aggressive tumor progression and to identify more specific and sensitive biomarkers for the diagnosis and targeted therapy of ESCC.

Nectin-2, also termed cluster of differentiation 112 and poliovirus receptor-related protein 2, in addition to Nectin-1, -3 and -4 , belongs to the Nectin family of cell adhesion molecules, which exert similar functions to immunoglobulin in the formation and continuation of tight junctions and adherence connections (6). Four members in this family have the same structure, containing extracellular loops, one transmembrane segment and a short cytoplasmic domain (7). Studies have reported the aberrant expression of Nectins and revealed their important roles in numerous cancer types (8-10). Notably, strong Nectin-2 expression was observed in leukemic blasts, myeloma, breast cancer, squamous cell and adenosquamous 
carcinomas, adenocarcinoma of gallbladder, colorectal carcinoma, pancreatic ductal adenocarcinomas and ovarian cancer, but its expression is reduced or absent in hepatocellular carcinoma (11-17). However, to the best of our knowledge, the involvement of Nestin-2 in ESCC remains unknown. The present study aimed to investigate the expression pattern of Nectin-2, its clinical significance and roles in the malignant phenotypes of ESCC.

\section{Materials and methods}

Patients and tissue samples. The Ethics Committees of People's Hospital of Lianshui County, People's Hospital of Xuyi County and Huai'an First People's Hospital (Huai'an, Jiangsu, China) approved the present study. All patients agreed to the procedure and signed consent forms.

A total of 106 patients with ESCC were diagnosed by endoscopic biopsy pathology between February 2006 and March 2012 at People's Hospital of Lianshui County, People's Hospital of Xuyi County and Huai'an First People's Hospital (Huai'an, Jiangsu, China). These patients were enrolled in the present study based on the following criteria: Newly diagnosed cancer of the esophagus without preoperative adjuvant chemotherapy or radiotherapy, and histologically-confirmed primary ESCC. There were 78 men and 28 women, ranging in age between 38 and 82 years (mean age, 50.6 years; median age, 50 years). The histological grade and clinical stage of the tumors were defined based on the UICC 2009 criteria (18). Of the 106 patients with ESCC, 16, 30 and 60 cases were classified as grades I-II, III and IV, respectively. In addition, 26, 30 and 50 cases were well, moderately and poorly differentiated, respectively. The clinicopathological characteristics of the 106 EOC patients are summarized in Table I. In total, 20 samples of normal esophageal mucosa were used as a control group. All specimens were collected from endoscopic biopsy, immediately snap-frozen in liquid nitrogen and maintained at $-80^{\circ} \mathrm{C}$ until use.

Cell culture and transfection. The human ESCC ECA109 and KYSE510 cell lines were purchased from the Chinese Academy of Sciences (Shanghai, China) and were cultured in RPMI-1640 medium (Gibco; Thermo Fisher Scientific, Inc., Waltham, MA, USA) supplemented with $10 \%$ fetal bovine serum (HyClone; GE Healthcare Life Sciences, Logan, UT, USA) and $100 \mathrm{U} / \mathrm{ml}$ penicillin/streptomycin (Gibco; Thermo Fisher Scientific, Inc.) and was maintained in a humidified $5 \%$ $\mathrm{CO}_{2}$ atmosphere at $37^{\circ} \mathrm{C}$.

The Nectin-2-targeted small interfering (si)RNA (si-Nectin-2; catalog no. sc-43169; sequence: GCCUUU UCUCAGACUCGATT) and negative control siRNA (si-con; catalog no. sc-44236; sequence: GACCAATCTCAGCAG ACAGTGCCATCATG) were purchased from Santa Cruz Biotechnology, Inc. (Dallas, TX, USA) and transfected into cells using Lipofectamine 2000 (Invitrogen; Thermo Fisher Scientific, Inc.), according to the manufacturer's protocol. After $48 \mathrm{~h}$, the subsequent experiments were performed.

Reverse transcription-quantitative polymerase chain reaction (RT-qPCR). RT-qPCR was performed to detect the expression level of Nectin-2 mRNA in ESCC tissues and normal esophageal mucosa, according to the protocol described in a previous study (19). GAPDH was used as an internal control. The qPCR primers were as follows: Nectin-2 forward, 5'-CGC CTCGATACCATGAGTT-3' and reverse, 5'-ACATCCCTG GCAGAAAGC-3'; and GAPDH forward5'-AGGTCGGTG TGAACGGATTTG-3' and reverse, 5'-GGGGTCGTTGAT GGCAACA-3'. The quantification cycle $(\mathrm{Cq})$ is defined as the fractional cycle number at which the fluorescence passes the fixed threshold. The relative expression of Nectin-2 mRNA was normalized to the expression of GAPDH mRNA using the $2^{-\Delta \Delta \mathrm{Cq}}$ method (20).

Western blot analysis. Western blot analysis was performed to detect the expression level of Nectin-2 protein in ESCC tissues and normal esophageal mucosa. Proteins in tissues and cell lysates were extracted using cell lysis buffer (Sigma-Aldrich; Merck KGaA, Darmstadt, Germany) and separated by $10 \%$ SDS-PAGE followed by electrotransference onto nitrocellulose membranes, which were then blocked with nonfat dry milk at room temperature for $1 \mathrm{~h}$. Subsequently, the membranes were incubated overnight at $4^{\circ} \mathrm{C}$, with the primary antibodies against Nectin-2 (dilution, 1:500; rabbit anti-human Nectin-2 antibody; cat. no. AF1166c; Abgent, Inc., San Diego, CA, USA) and GAPDH (dilution, 1:2,000; cat. no. SAB168068; Sigma-Aldrich; Merck KGaA). Subsequently, the membranes were washed with TBST several times and then incubated with horseradish peroxidase-conjugated secondary antibodies (dilution, 1:3,000; cat. no. SAB186192; Sigma-Aldrich; Merck $\mathrm{KGaA}$ ) at room temperature for $1 \mathrm{~h}$. Immunoblots were visualized by the enhanced chemiluminescence detection system (Pierce; Thermo Fisher Scientific, Inc.).

Immunohistochemistry. Immunohistochemistry was performed to detect the expression pattern and subcellular localization of Nectin-2 protein in 106 ESCC tissues and 20 normal esophageal mucosa. All tissue samples were fixed with $4 \%$ formaldehyde, embedded in paraffin, and sectioned into $4-\mu \mathrm{m}$ slices. The sections were deparaffinized in xylene and rehydrated in graded ethanol. Endogenous peroxidase activity was blocked for $15 \mathrm{~min}$ with $3 \%$ hydrogen peroxide in phosphate-buffered saline (PBS). The sections were incubated with primary rabbit anti-human Nectin-2 antibody (dilution, 1:500; cat. no. AF1166c; Abgent, Inc.) overnight at $4^{\circ} \mathrm{C}$ and then incubated with IgG-horseradish peroxidase (dilution, 1:1,000; cat. no. ZS6061; Zhongshan Biotechnology, Co., Ltd., Zhongshan, China) for $1 \mathrm{~h}$ at $37^{\circ} \mathrm{C}$. Immunostaining was performed using the avidin biotin peroxidase complex method and antigen-antibody reactions were visualized with the chromogen diaminobenzidine.

To evaluate the results of immunohistochemistry, the sections were observed under optical microscopy (x10 and $\mathrm{x} 200$ ) by two pathologists who were blinded to the clinicopathological characteristics of the 106 patients with ESCC. The immunoreactive score (IRS) was calculated by multiplying the intensity score and the percentage score of immunostaining. The former was defined as follows: 3 , strong; 2 , moderate; 1 , weak; and 0 , negative. The latter was defined as follows: 0 , $<5 \%$ of cells; $1,5-25 \% ; 2,26-50 \% ; 3,50-75 \%$; and $4,>75 \%$. The median IRS value (3.9) of Nectin-2 protein was used as a cutoff point to divide all 106 patients with ESCC into Nectin-2-high $(n=55)$ and Nectin-2-low $(n=51)$ groups. 
Table I. Association between Nectin-2 and various clinicopathological features of patients with esophageal squamous cell carcinoma.

\begin{tabular}{|c|c|c|c|c|}
\hline \multirow{2}{*}{$\begin{array}{l}\text { Clinicopathological } \\
\text { features }\end{array}$} & \multirow[b]{2}{*}{ Cases, $\mathrm{n}$} & \multicolumn{2}{|c|}{$\begin{array}{c}\text { Nectin-2 } \\
\text { expression, } \mathrm{n}(\%)\end{array}$} & \multirow[b]{2}{*}{ P-value } \\
\hline & & High & Low & \\
\hline Age & & & & NS \\
\hline$<60$ years & 40 & $20(50.0)$ & $20(50.0)$ & \\
\hline$\geq 60$ years & 66 & $35(53.0)$ & $31(47.0)$ & \\
\hline Gender & & & & NS \\
\hline Male & 70 & 37 (52.9) & $33(47.1)$ & \\
\hline Female & 36 & $18(50.0)$ & $18(50.0)$ & \\
\hline Differentiation & & & & 0.02 \\
\hline Well-moderate & 56 & $20(35.7)$ & $36(64.3)$ & \\
\hline Poor & 50 & $35(70.0)$ & $15(30.0)$ & \\
\hline Tumor size & & & & NS \\
\hline$<5 \mathrm{~cm}$ & 46 & $25(54.3)$ & $21(45.7)$ & \\
\hline$\geq 5 \mathrm{~cm}$ & 60 & $30(50.0)$ & $30(50.0)$ & \\
\hline TNM stage & & & & 0.006 \\
\hline I-II & 16 & $1(6.3)$ & $15(93.7)$ & \\
\hline III & 30 & $9(30.0)$ & $21(70.0)$ & \\
\hline IV & 60 & $45(75.0)$ & $15(75.0)$ & \\
\hline
\end{tabular}

NS, no significant difference; TNM, tumor-node-metastasis.

Cell migration and invasion assays. Transwell assay was performed to assess the migration and invasion abilities of ESCC cells transfected with si-Nectin-2 or si-con using a modified two-chamber migration assay with a pore size of $8 \mu \mathrm{m}$ (Merck KGaA).

For the migration assay, $1 \times 10^{5}$ cells were suspended with serum-free RPMI-1640 (Gibco; Thermo Fisher Scientific, Inc.) and seeded in the upper chambers of the 24-well Transwell plates. The lower well of the chamber was supplemented with RPMI-1640 containing 10\% serum (Gibco; Thermo Fisher Scientific, Inc.). Following incubation for $24 \mathrm{~h}$, any cells remaining on top of the insert were removed by scraping and the migrating cells on the underside of the membrane were fixed with $4 \%$ formaldehyde, stained with $0.5 \%$ crystal violet, and examined under an optical microscope (magnification, x10 and x200; Olympus Corporation, Tokyo, Japan) in 10 randomly selected fields.

For the invasion assay, $1 \times 10^{5}$ cells were suspended with serum-free RPMI-1640 (Gibco; Thermo Fisher Scientific, Inc.) and seeded in the upper chambers of the 24-well Transwell chambers coated with Matrigel (1 mg/ml; BD Biosciences, Franklin Lakes, NJ, USA). The lower chamber was supplemented with RPMI-1640 containing 10\% serum (Gibco; Thermo Fisher Scientific, Inc.) as a chemoattractant. Subsequent to incubation for $24 \mathrm{~h}$, the invading cells on the underside of the membrane were fixed with $4 \%$ formaldehyde, stained with $0.5 \%$ crystal violet, and examined under an optical microscope (magnification, x10 and x200; Olympus Corporation) in 10 randomly selected fields.
Statistical analysis. All statistical analyses in the current study were conducted by using SPSS version 11.0 for Windows (SPSS Inc., Chicago, IL, USA). Each experiment was performed in triplicate. Data were expressed as the mean \pm standard error of three repeated assays. Differences between various groups were assessed using Student's t-test. Fisher's exact test and $\chi^{2}$ were performed to assess the associations between Nectin-2 expression and different clinicopathological characteristics. $\mathrm{P}<0.05$ was considered to indicate a statistically significant difference.

\section{Results}

Elevated Nectin-2 expression in human ESCC tissues. RT-qPCR showed that the relative expression of Nectin-2 mRNA in ESCC tissues was significantly elevated compared with that in the normal esophageal mucosa (ESCC vs. normal, $4.09 \pm 1.37$ vs. $2.00 \pm 0.67$; $\mathrm{P}<0.001$; Fig. 1A). Similarly, western blot analysis also found an increased expression level ofNectin-2 protein in ESCC tissues compared with normal esophageal mucosa (ESCC vs. normal, 3.93 \pm 1.40 vs. $1.76 \pm 0.57$; $\mathrm{P}=0.003$; Fig. $1 \mathrm{~B})$.

In addition, the expression pattern and subcellular localization of Nectin-2 protein in ESCC and normal esophageal mucosa was further examined by immunohistochemistry. As shown in Fig. 1C, positive staining of Nectin-2 protein was localized in the cytoplasm of cancer cells in ESCC tissues. Of the 106 ESCC tissues, 38 (35.8\%) and 42 (39.6\%), respectively, demonstrated strong and moderate positive immunoreactivity for the Nectin-2 protein, whereas $16(15.1 \%)$ showed a low Nectin-2 expression and 10 (9.4\%) were Nectin-2-negative. By contrast, all normal esophageal mucosa were weakly or not immunostained for Nectin-2 expression. Additionally, the statistical analysis demonstrated that the IRS of Nectin-2 protein in the ESCC tissues was significantly increased compared with normal esophageal mucosa (ESCC vs. normal, $3.96 \pm 1.41$ vs. $1.92 \pm 1.87$; $\mathrm{P}=0.005$; Fig. $1 \mathrm{D})$, which was consistent with the results of RT-qPCR and western blot analysis.

Elevated expression of Nectin-2 protein is associated with aggressive progression of ESCC patients. Table I summarized the association of Nectin-2 protein expression with the clinicopathological parameters of ESCC patients. There were significant associations between Nectin-2 protein expression and clinicopathological parameters, such as age, gender and tumor size $(\mathrm{P}>0.05)$. Conversely, significant associations were observed between Nectin-2 protein expression, and tumor stage and differentiation in ESCC patients. The average IRS of Nectin-2 protein in ESCC tissues obtained from patients with advanced tumor stage $(\mathrm{P}=0.006$; Table $\mathrm{I})$ and poor differentiation ( $\mathrm{P}=0.02$; Table $\mathrm{I})$ was increased compared with tissues obtained from patients with early tumor stage and well-moderate differentiation.

Loss of Nectin-2 protein expression inhibited ESCC cell migration and invasion in vitro. To elucidate the involvement of Nectin-2 in ESCC cell migration and invasion abilities, Nectin-2 siRNA was used to knockdown the expression of Nectin-2 in the human ESCC ECA109 and KYSE510 cell lines. As shown in Fig. 2, the relative protein expression of Nectin-2 


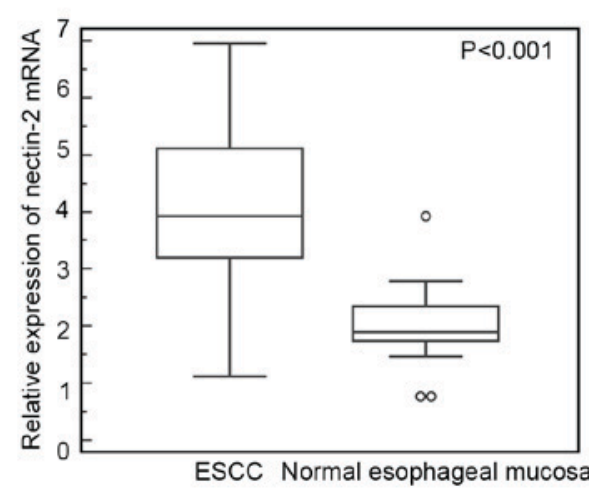

$\mathrm{C}$

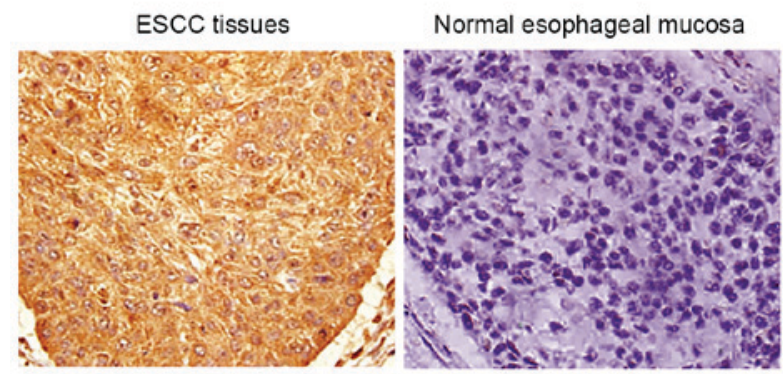

B

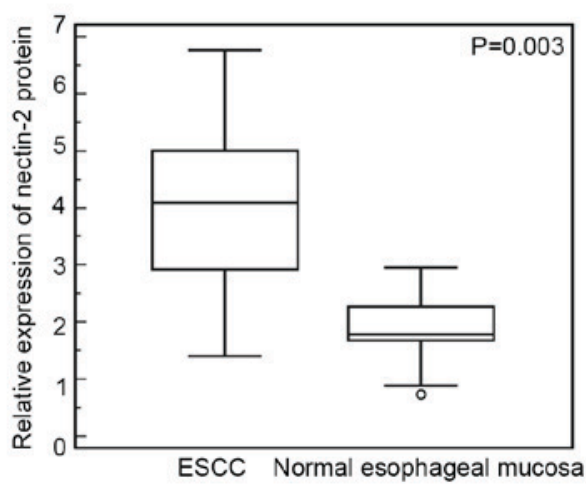

D

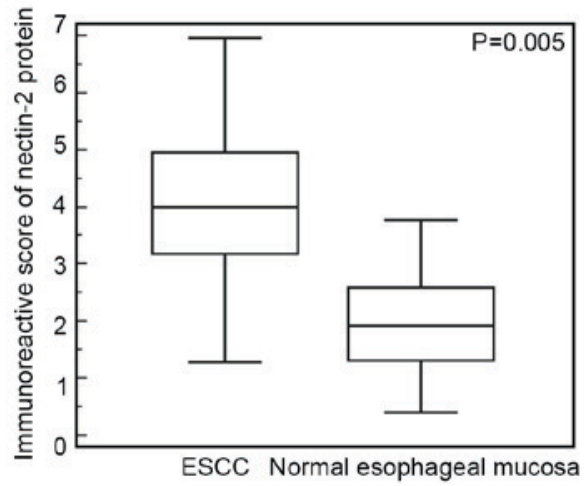

Figure 1. Elevated expression of Nectin-2 mRNA and protein in human ESCC tissues. (A) Relative expression of Nectin-2 mRNA in ESCC tissues was significantly elevated compared with that in the normal esophageal mucosa (ESCC vs. normal, $4.09 \pm 1.37$ vs. $2.00 \pm 0.67$; $\mathrm{P}<0.001$ ). (B) Western blot analysis found increased Nectin-2 protein expression in ESCC tissues compared with normal esophageal mucosa (ESCC vs. normal, 3.93 \pm 1.40 vs. 1.76 $\pm 0.57 ; \mathrm{P}=0.003$ ) (C) Positive staining of Nectin-2 protein was localized in the cytoplasm of cancer cells in ESCC tissues. (D) Statistical analysis demonstrated that the immunoreactive score of Nectin-2 protein in the ESCC tissues was significantly higher than that in the normal esophageal mucosa (ESCC vs. Normal, $3.96 \pm 1.41$ vs. $1.92 \pm 1.87 ; \mathrm{P}=0.005)$. ESCC, esophageal squamous cell carcinoma.

in ECA109 and KYSE510 cells was markedly decreased by transfection with Nectin-2 siRNA $(\mathrm{P}<0.001)$. The migration and invasion abilities of ECA109 and KYSE510 cells transfected with Nectin-2 siRNA were suppressed compared with the two control groups $(\mathrm{P}<0.05$; Fig. 3).

\section{Discussion}

Metastasis is a complex process of cancer cell migration and invasion to new tissues, and has been recognized as the principal cause of mortality in ESCC patients $(21,22)$. Thus, it is important to better understand the mechanisms underlying the carcinogenesis and to identify an indicator capable of predicting the malignant potential of ESCC. In the current study, the data illustrated the expression patterns of Nectin-2 in ESCC tissues, followed by demonstrating the association between the Nectin-2 expression and clinicopathological parameters based on 106 clinical samples, and identified the role of Nectin-2 in malignant phenotypes of ESCC cells. Differential expression of Nectin-2 at mRNA and protein levels was measured among primary ESCC tissues and normal esophageal mucosa by RT-qPCR, western blot analysis and immunohistochemistry, which all showed the upregulation of Nectin-2 in ESCC tissues compared with normal esophageal mucosa. Nectin-2 upregulation was then found to be closely correlated with advanced tumor stage and poor differentiation. Additional in vitro experiments presented here showed that the
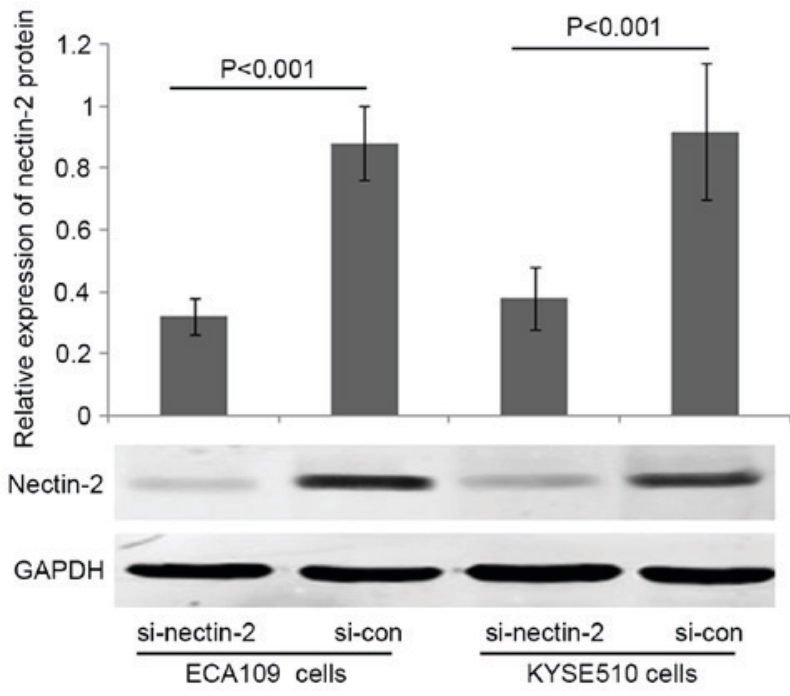

Figure 2. Loss of Nectin-2 protein expression in the human esophageal squamous cell carcinoma ECA109 and KYSE510 cell lines subsequent to transfection with Nectin-2 siRNA in vitro. siRNA, small interfering RNA; si-nectin-2, siRNA targeting Nectin-2; si-con, control siRNA.

two human ESCC cell lines transfected with Nectin-2 siRNA had reduced migration and invasion abilities. These findings suggested that Nectin-2 could play a vital role in the malignant progression of ESCC. 
A

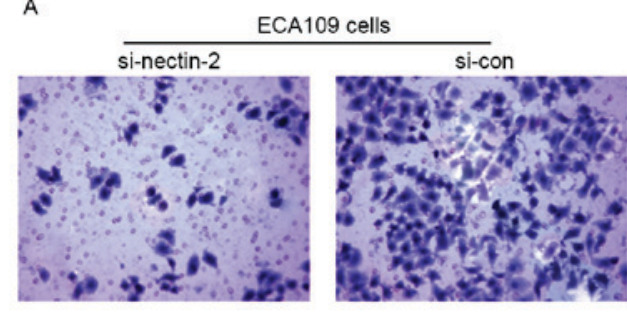

KYSE 510 cells
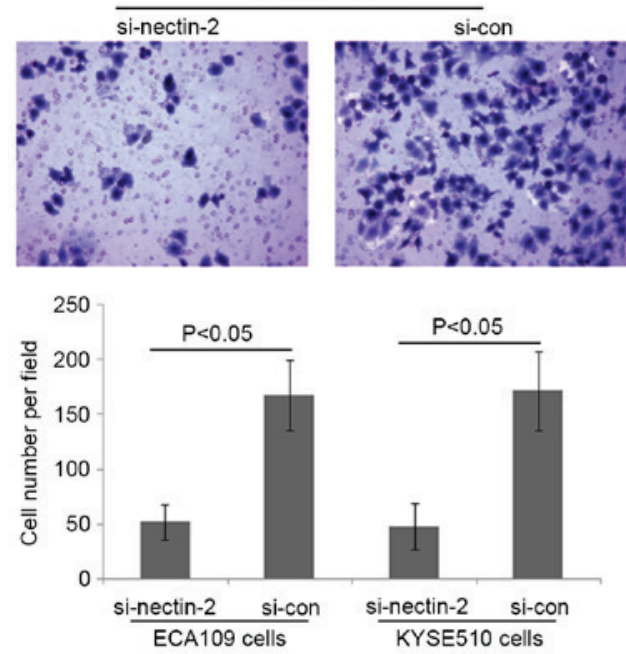

B

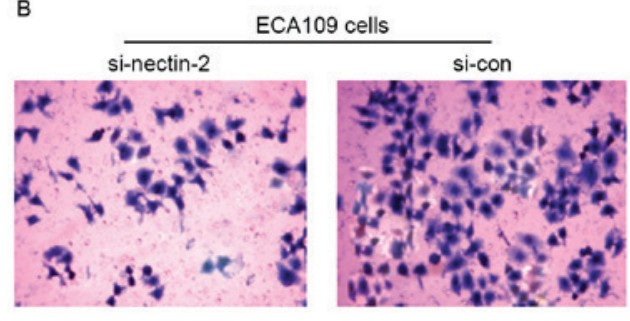

KYSE510 cells
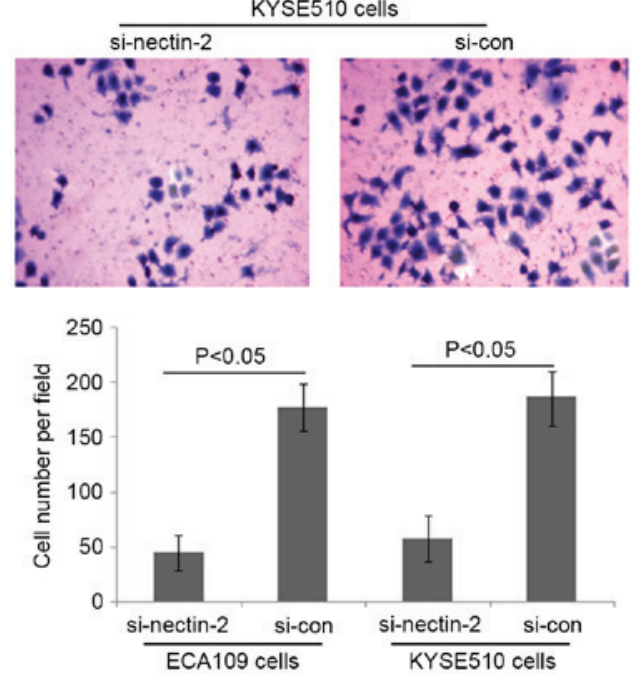

Figure 3. Loss of Nectin-2 protein expression inhibits esophageal squamous cell carcinoma cell migration and invasion in vitro. (A) The migration abilities of ECA109 and KYSE510 cells transfected with Nectin-2 siRNA were suppressed compared to the two control groups (both P<0.05). (B) The invasion abilities of ECA109 and KYSE510 cells transfected with Nectin-2 siRNA were suppressed compared to the two control groups (both P<0.05). siRNA, small interfering RNA; si-nectin-2, siRNA targeting Nectin-2; si-con, control siRNA.

Nectin-2 belongs to the Nectin family of $\mathrm{Ca}^{2+}$-independent immunoglobulin-like cell adhesion molecules and functions as a junction molecule regulating the cell adhesion between epithelial cells by creating trans-dimmers with neighbor cells of Nectin-2 member (23). It is ubiquitously expressed in cells of various tissues, including neuronal, hematopoietic, endothelial and epithelial cells (24). Nectin-2 has been concerned in different diseases in humans, and has currently been defined as a marker, and potential therapeutic target in numerous types of cancers. Nectin-2 is often overexpressed in cancer cells, and is associated with a poor prognosis. For example, Karabulut et al (13) performed ELISA and determined that the serum Nectin-2 levels in 140 colorectal cancer patients were dramatically compared with patients in the healthy control group. The diagnostic and prognostic roles of serum levels of Nectin-2 in patients with colorectal cancer were also reported. Miao et al (14) identified Nectin-2 as a biomarker for metastasis and poor prognosis of squamous cell/adenosquamous carcinomas and adenocarcinoma of the gallbladder. Oshima et al (15) observed the overexpression of Nectin-2 in breast and ovarian cancers and anti-tumor activity of anti-Nectin-2 monoclonal antibodies via strong antibody-dependent cellular cytotoxicity, indicating its potential as a target for antibody therapy against breast and ovarian cancers. Liang et al (16) reported that positive Nectin-2 expression was associated with progression and poor prognosis in pancreatic ductal adenocarcinoma patients. By contrast, Huang et al (17) found that Nectin-2 expression was significantly reduced in hepatocellular carcinoma tissues, as well as the majority of hepatocellular carcinoma cell lines, as determined by western blot analysis and immunofluorescence analyses. This study also confirmed that the downregulation of Nectin-2 may be an important mechanism through which hepatocellular carcinoma cells evade the natural killer cell-mediated immunosurveillance, and may function as a marker for poor prognosis in patients with this malignancy (17). These data suggest that Nectin-2 may function as either an oncogene or a tumor suppressor in various cancers.

The present study evaluated the expression of Nectin-2 in 106 ESCC tissues at the mRNA and protein levels. The results showed that Nectin-2 expression at the mRNA and protein levels was evidently increased in human ESCC tissues compared with adjacent normal esophageal tissues. In addition, consistent with the results of studies in other types of cancers $(14,17)$, the immunohistochemistry analysis demonstrated that staining for Nectin-2 was present in the cytoplasm of the cancer cells. Consequently, it was hypothesized that overexpression of Nectin-2 plays an important role in the progression of ESCC. Since tumor cell motility is a sign of invasiveness and an essential step in metastasis, the function of Nectin-2 in ECA109 and KYSE510 cells was investigated using a gene knockdown technique. The present study showed that the migration and invasion of the ESCC ECA109 and KYSE510 cell lines subsequent to transfection with siRNA specific to Nectin-2 were evidently reduced compared to the negative control cells, indicating that Nectin-2 may act as an oncogene for promoting cell migratory ability and invasiveness in ESCC progression. 
In conclusion, to the best of our knowledge, the present data revealed for the first time that Nectin-2 is overexpressed in ESCC and associated with aggressive cancer progression. The present data also indicated that the silencing of Nectin-2 with siRNA in ESCC cells may inhibit malignant biological properties in cells, indicating potential as a possible marker or therapeutic target for ESCC. Additional studies are required to elucidate the mechanisms underlying the functions of Nectin-2 as a useful marker for ESCC.

\section{References}

1. Baba Y, Watanabe M, Yoshida N and Baba H: Neoadjuvant treatment for esophageal squamous cell carcinoma. World J Gastrointest Oncol 6: 121-128, 2014.

2. Shang L and Wang M: Molecular alterations and clinical relevance in esophageal squamous cell carcinoma. Front Med 7: 401-410, 2013.

3. Yang CS, Chen X and Tu S: Etiology and prevention of esophageal cancer. Gastrointest Tumors 3: 3-16, 2016.

4. Li JS, Ying JM, Wang XW, Wang ZH, Tao Q and Li LL: Promoter methylation of tumor suppressor genes in esophageal squamous cell carcinoma. Chin J Cancer 32: 3-11, 2013.

5. Lehrbach DM, Nita ME and Cecconello I: Molecular aspects of esophageal squamous cell carcinoma carcinogenesis. Arq Gastroenterol 40: 256-261, 2003.

6. Devilard E, Xerri L, Dubreuil P, Lopez M and Reymond N: Nectin-3 (CD113) interacts with Nectin-2 (CD112) to promote lymphocyte transendothelial migration. PLoS One 8: e77424, 2013.

7. Zhang X and Lui WY: Dysregulation of nectin-2 in the testicular cells: An explanation of cadmium-induced male infertility. Biochim Biophys Acta 1839: 873-884, 2014.

8. Liu J, Qian X, Chen Z, Xu X, Gao F, Zhang S, Zhang R, Qi J, Gao GF and Yan J: Crystal structure of cell adhesion molecule nectin-2/CD112 and its binding to immune receptor DNAM-1/CD226. J Immunol 188: 5511-5520, 2012.

9. Mandai K, Rikitake Y, Mori M and Takai Y: Nectins and nectin-like molecules in development and disease. Curr Top Dev Biol 112: 197-231, 2015 .

10. Samanta D and Almo SC: Nectin family of cell-adhesion molecules: Structural and molecular aspects of function and specificity. Cell Mol Life Sci 72: 645-658, 2015.

11. Pende D, Spaggiari GM, Marcenaro S, Martini S, Rivera P, Capobianco A, Falco M, Lanino E, Pierri I, Zambello R, et al: Analysis of the receptor-ligand interactions in the natural killer-mediated lysis of freshly isolated myeloid or lymphoblastic leukemias: Evidence for the involvement of the Poliovirus receptor (CD155) and Nectin-2 (CD112). Blood 105: 2066-2073, 2005.

12. Wu MR, Zhang T, Alcon A and Sentman CL: DNAM-1-based chimeric antigen receptors enhance $\mathrm{T}$ cell effector function and exhibit in vivo efficacy against melanoma. Cancer Immunol Immunother 64: 409-418, 2015.
13. Karabulut M, Gunaldi M, Alis H, Afsar CU, Karabulut S, Serilmez M, Akarsu C, Seyit H and Aykan NF: Serum nectin-2 levels are diagnostic and prognostic in patients with colorectal carcinoma. Clin Transl Oncol 18: 160-171, 2016.

14. Miao X, Yang ZL, Xiong L, Zou Q, Yuan Y, Li J, Liang L, Chen $\mathrm{M}$ and Chen S: Nectin-2 and DDX3 are biomarkers for metastasis and poor prognosis of squamous cell/adenosquamous carcinomas and adenocarcinoma of gallbladder. Int J Clin Exp Pathol 6: 179-190, 2013

15. Oshima T, Sato S, Kato J, Ito Y, Watanabe T, Tsuji I, Hori A, Kurokawa $\mathrm{T}$ and Kokubo T: Nectin-2 is a potential target for antibody therapy of breast and ovarian cancers. Mol Cancer 12: 60, 2013.

16. Liang S, Yang Z, Li D, Miao X, Yang L, Zou Q and Yuan Y: The clinical and pathological significance of Nectin-2 and DDX3 expression in pancreatic ductal adenocarcinomas. Dis Markers 2015: 379568, 2015.

17. Huang X, Qu P, Chen Y, Zhou X, Wu Y, Liu F, Wang D, Zhang J and An J: Low expression of CD112 is associated with poor overall survival in patients with hepatocellular carcinoma. Hum Pathol 45: 1944-1950, 2014.

18. Yamasaki M, Miyata H, Miyazaki Y, Takahashi T, Kurokawa Y, Nakajima K, Takiguchi S, Mori M and Doki Y: Evaluation of the nodal status in the 7th edition of the UICC-TNM classification for esophageal squamous cell carcinoma: Proposed modifications for improved survival stratification: Impact of lymph node metastases on overall survival after esophagectomy. Ann Surg Oncol 21: 2850-2856, 2014

19. Chen G, Peng J, Zhu W, Tao G, Song Y, Zhou X and Wang W: Combined downregulation of microRNA-133a and microRNA-133b predicts chemosensitivity of patients with esophageal squamous cell carcinoma undergoing paclitaxel-based chemotherapy. Med Oncol 31: 263, 2014.

20. Livak KJ and Schmittgen TD: Analysis of relative gene expression data using real-time quantitative PCR and the 2(-Delta Delta C(T)) method. Methods 25: 402-408, 2001.

21. Chen MQ, Xu BH and Zhang YY: Analysis of prognostic factors for esophageal squamous cell carcinoma with distant organ metastasis at initial diagnosis. J Chin Med Assoc 77: 562-566, 2014.

22. Salian V, Dinakar C, Shetty P and Ajila V: Etiological trends in oral squamous cell carcinoma: A retrospective institutional study. Cancer Transl Med 2: 33-36, 2016.

23. Sakisaka T and Takai Y: Biology and pathology of nectins and nectin-like molecules. Curr Opin Cell Biol 16: 513-521, 2004

24. Sakisaka T, Ikeda W, Ogita H, Fujita N and Takai Y: The roles of nectins in cell adhesions: Cooperation with other cell adhesion molecules and growth factor receptors. Curr Opin Cell Biol 19: 593-602, 2007.

This work is licensed under a Creative Commons Attribution-NonCommercial-NoDerivatives 4.0 International (CC BY-NC-ND 4.0) License. 